EXEMPLARIa Classica

Journal of Classical Philology

21, 2017, pp. 25-42

ISSN 1699-3225

\title{
VIRGILIO (ENEIDA) Y OVIDIO (METAMORFOSIS): DOS TRANSMISIONES TEXTUALES DISIMÉTRICAS ${ }^{1}$
}

\author{
ÁNGEL EsCOBAR \\ Universidad de Zaragoza \\ aescobar@unizar.es
}

\section{SUMMARY}

Although the respective textual transmissions of Virgil's Aeneid and Ovid's Metamorphoses share some similarities, there are also some clearly distinctive features, which allow us to qualify them as 'asymmetric' textual traditions. However, this does not imply that a specific methodological approach must be adopted in order to study the textual variation in each tradition, nor to establish each text.

KEY WORDS

Aeneis; Metamorphoses; textual transmission.

\section{RESUMEN}

Pese a ofrecer algunos elementos comunes, las transmisiones textuales de las obras mayores de Virgilio (Aen.) y de Ovidio (Met.) presentan una serie de rasgos distintivos, de carácter objetivo y muy marcados, que permiten calificarlas - desde un punto de vista tipológico- como 'disimétricas', pero que, sin embargo, no parecen bastar para hacerlas susceptibles de un análisis de variantes diferenciado desde el punto de vista metodológico y con utilidad práctica para la fijación del texto.

Palabras Clave

Eneida; Metamorfosis; transmisión textual.

Fecha de recepción: 27/01/2017

Fecha de aceptación y versión final: 18/09/2017

${ }^{1}$ Agradecemos a los evaluadores de nuestro artículo sus valiosas sugerencias, que hemos procurado recoger en la versión definitiva. Todos los enlaces electrónicos citados se encuentran operativos a fecha de 16/09/2017. 


\section{Primeras FASES EN LA TRANSMisión}

Desde una perspectiva histórico-textual, resulta posible analizar el contraste tipológico existente entre transmisiones diversas, aun cuando cada una de ellas posea una idiosincrasia muy marcada, tanto en el ámbito de los textos griegos como en el de los textos latinos. En el caso de dos autores clásicos tan relativamente próximos en el tiempo y tan difundidos en todo momento como Virgilio y Ovidio, esa comparación tipológica nos parece muy pertinente, y no sólo a causa de los dos motivos recién mencionados. Es así sobre todo en lo que se refiere a sus respectivas obras más extensas (ambas "épicas", según la etiqueta tradicional), Eneida y Metamorfosis, muy distintas en intereses e incluso antagónicas en muchos aspectos, si bien ha solido considerarse que el maius opus de Ovidio (Trist. 2.63: inspice maius opus, quod adhuc sine fine reliqui) era sobre todo una particular secuela del de Virgilio, su más "admirado" referente (Trist. 4.10.51: Vergilium uidi tantum²).

Las concomitancias entre ambas transmisiones se han señalado con frecuencia, destacándose los reflejos que parece ofrecer la tradición de Metamorfosis respecto a su precedente virgiliano. A la luz de esta clave ha solido interpretarse, sobre todo, la supuesta quema - casi 'cremación'- del original de Metamorfosis por parte de Ovidio en el momento de su exilio ${ }^{3}$, urdida probablemente para emular y "realizar" en cierto modo el deseo virgiliano de entregar al fuego una obra imperfecta (Vita Donati, cap. 39 Hardie): si la muerte impidió a Virgilio concluir, revisar y publicar o editar su Eneida

${ }^{2}$ Citamos por J. B. Hall, P. Ovidi Nasonis Tristia, Stuttgart - Leipzig 1995. En general, cf. R. F. Thomas, "Ovid's reception of Virgil”, en P. E. Knox, ed., A companion to Ovid, Chichester 2009, 294-5, donde incluso se secunda la audaz hipótesis de que el Ars amatoria sea correlato de Georgica. Buena prueba del carácter modélico de Virgilio son, sin duda, las muchas parodias ovidianas (R. J. Tarrant, "Ovid and ancient literary history", en Ph. Hardie, ed., The Cambridge companion to Ovid, Cambridge 2002, 24, Thomas, "Ovid's reception", 298, así como 306, con referencia a Met. como 'palimpsesto' virgiliano, al igual que ocurre con Tristia: J. P. Bews, "The metamorphosis of Virgil in the Tristia of Ovid", BICS 31, 1984, 51-60; sobre Virgilio como lifetime engagement para Ovidio, en general, cf. S. Myers, "The metamorphosis of a poet: recent work on Ovid", JRS 89, 1999, 195-6).

${ }^{3}$ Cf. Trist. 1.7.15-6 (haec [sc. carmina] ego discedens, sicut bene multa meorum, / ipse mea posui maestus in igne manu), 19-20 (sic ego, non meritos, mecum peritura, libellos, / inposui rapidis uiscera nostra rogis), así como, ya con carácter genérico, tres lugares más: 4.1.101-2 (saepe manus demens, studiis irata malignis, / misit in arsuros carmina nostra focos), 10.61-4 (multa quidem scripsi, sed quae uitiosa putaui, / emendaturis ignibus ipse dedi. I tum quoque, cum fugerem, quaedam placitura cremaui, / iratus studio carminibusque meis), 5.12.61-8; según F. K. A. Martelli (Ovid's revisions: the editor as author, Cambridge 2013, 52), "References to book-burning will come to prominence later on in Ovid's literary career, and with particular frequency in the Tristia, where the poet uses them to mark how far his estimation of the quality of his literary output in exile has sunk"; otras referencias a la naturaleza "póstuma" de Metamorfosis en Trist.1.1.117-8 (sunt quoque mutatae, ter quinque uolumina, formae, / nuper ab exequiis pignora [Hall, carmina codd.] rapta meis) y 3.14.19-20 (sint quoque mutatae, ter quinque uolumina, formae, I pignora [Hall, carmina codd.] de domini funere rapta sui). 
completa, el destierro ovidiano habría tenido un efecto similar sobre el texto de Metamorfosis, arrancado de su autor y pasto efectivo esta vez de las llamas. Naturalmente, debe prestarse muy escasa credibilidad a esta declaración de Tristia ${ }^{4}$; su carácter ficticio, histriónico y meramente simbólico se corrobora no sólo por la inutilidad en sí que habría caracterizado tal acto (siendo que la obra ya circulaba quizá por Roma: Trist.1.7.24), sino también por otros factores, como el carácter autocomplaciente del autor, tan amante de su ingenio o talento (Quint. 10.1.88 y 98) e incluso de sus propios defectos (Séneca el Viejo, Contr. 2.2.12, 9.5.17). Que Ovidio adobe el relato representándose a sí mismo como "infanticida" de sus propias entrañas, identificadas con su adhuc crescens et rude carmen (Trist. 1.7.22), mediante ecos verbales tomados quizá -en parte- del relato sobre Meleagro en Met. 8.445-525 (cf. v. 478: conualuit, 'rogus iste cremet mea uiscera' dixit $t^{5}$ ), no hace sino incidir, mediante una poderosa imagen, en uno de sus temas literarios recurrentes $^{6}$.

Una vez "recuperado" el texto gracias a los varios exempla subsistentes ${ }^{7}$ y que podrían haberlo dado a conocer antes de ser 'editado' (Trist. 1.7.24:

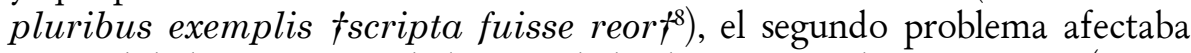
a su calidad misma, por haber quedado desprovisto de emendatio (Trist. 1.7.40: emendaturus, si licuisset, erat), esto es, como opus incorrectum e inmerecedor de renombre (Trist. 3.14.23: nunc incorrectum populi perue-

4 El toque "realista" que enfatizó $H$. Magnus es muy débil argumento ("Ovids Metamorphosen, in Doppelter Fassung?", Hermes 40, 1905, 192: "[...] die Sache durch den Zusatz sicut bene multa meorum [Trist.1.7.15] auf realen Boden gerückt wird und es an sich nicht unwahrscheinlich ist, daß einer, der auf Nimmerwiederkehr geht, allerhand Scripturen ins Feuer wirft").

${ }^{5}$ S. Hinds, "Booking the return trip: Ovid and Tristia I", en P. E. Knox, ed., Oxford readings in Ovid, Oxford - Nueva York 2009, 430.

${ }^{6}$ Como es el del aborto furtivo, doblemente criminal por perpetrarse a ocultas y en silencio (en relación sobre todo con Her. 11, cf. Á. Escobar, "Oppressed voice and oppressing silence: some ancient attitudes towards abortion and infanticide", Euphrosyne 40, 2012, 109-22); a las referencias aportadas por Hinds (cit. en nota anterior) pueden añadirse quizá las de Am. 2.14.25 (sine crescere nata) y 27 (uestra quid effoditis subiectis uiscera telis), Her. 11.50 (rudis ad partus), 92 (uiscera).

7 "[...] presumably in the private libraries of friends and the librarii who keep these putative copies", en opinión de L. Jansen, "Modern covers and paratextual strategy in Ovidian elegy", en The Roman paratext: frame, texts, readers, Cambridge 2014, 274-5.

${ }^{8}$ Desde el punto de vista de la dicción cabría comparar Her. 20.242 (quae fuerint in eo scripta fuisse rata), sin que ello aporte luz alguna; el fuisse meis del vetustissimus Marc. 223 sugiere resolver una posible abreviatura inicial en el sencillo fuere meis (cf. por ejemplo Her. 10.38: uerbera cum uerbis mixta fuere meis), en oración también parentética (pluribus exemplis scripta fuere meis); exemplum ("muestra", "espécimen" en nuestro texto) se consagrará con el significado técnico de "texto" - frente a exemplar- más tarde (así en la famosa subscripción liviana de Nicomachus Dexter a inicios del siglo V: emendaui ad exemplum parentis mei; cf. J. E. G. Zetzel, "Emendavi ad Tironem: some notes on scholarship in the second century A. D.”, HSCPh 77, 1973, 227). 
nit in or $^{9}$ ), condición que también lo aproximaba en cierto modo a su correlato épico virgiliano. Según observó Hartman con toda razón, cabe pensar que las enmiendas que Ovidio consideraba necesarias afectarían al contenido literario -e incluso ideológico, probablemente- de la obra, y no tanto a erratas o aspectos puramente versificatorios y formales ${ }^{10}$; creemos que así lo sugiere también el casi hápax incorrectum, que remite sobre todo, dentro de la tradición retórica, al ámbito de la corrección "de pensamiento" de Metamorfosis - a diferencia del de Eneida, con sus uersus trunci o truncati- no ofrece signos evidentes de que llegara a publicarse inacabado o insuficientemente limado, lo que invita a sospechar que el autor, reluctante a dar sus obras por concluidas ${ }^{12}$, se limitaba a recurrir también en este caso a un

${ }^{9}$ Cf. Trist.1.7.27-30 (nec tamen illa legi poterunt patienter ab ullo, / nesciet extremam siquis abesse manum. I ablatum mediis opus est incudibus illud, / defuit et coeptis ultima lima meis), 2.63-4 (inspice maius opus, quod adhuc sine fine reliqui [frente al tenetur de M, con J. Diggle, "Notes on Ovid's Tristia, books I-II", CQ 30, 1980, 412-3], I in non credendos corpora uersa modos [del. Bentley]), pese a las diversas interpretaciones posibles del sine fine, 555-556 (coeptaque [Hall, frente al transmitido y seguramente correcto dictaque: P. Knox, res. de Hall, P. Ovidi Nasonis Tristia, en < http://bmcr.brynmawr. edu/1997/97.03.31.html >] sunt nobis, quamuis manus ultima coeptis / defuit, in facies corpora uersa nouas), 3.14.19-24 (sint quoque mutatae, ter quinque uolumina, formae, / pignora de domini funere rapta sui. / illud opus potuit, si non prius ipse perissem, I certius a summa nomen habere manu: / nunc incorrectum populi peruenit in ora, / in populi quicquam si tamen ore meum est).

${ }^{10}$ J. J. Hartman, "De Ovidii Metamorphosesin edendis", Mnem. 18, 1890, 179 ("Quid in suo poemate emendaturum, si licuisset, fuisse Ovidium censeamus? Unam alteramque, ut opinor, fabulam in brevius contraxisset, e rebus mythologicis, quas hic illic permultas sine ullo ornamento poetico enumeravit [exemplo sit locus VII, 354-400] paucas elegisset quas fusius elaboraret, fortasse etiam nonnumquam e duobus locis parallelis alterum paulo aliter enuntiasset, postremo, [...] interdum unum versum delevisset vel e duobus fecisset unum, vel pro versu versusve parte paulo languidiore verba diserta magis magisque acuta posuisset), 182 (Sed numquam adducar ut credam - id quod nonnulli editores sibi persuadere videntur- poëtam fatendo ultimam libro defuisse manum significasse in eo remansisse aliquot versus inepte factos vitiososque [...] Si quid ergo in Metamorphosesin legitur quod sit contortum, obscurum, absurdum, quodve a sermonis usu nimis recedat vel improbabilem requirat explicationem, id ego invito poëta quin natum sit minime dubito"). Lo contrario sugería, pensando quizá en el more ursino virgiliano pero sin mayor fundamento aparente, Ch. E. Murgia, "Ovid Met. 1.544-547 and the theory of double recension", Classical Antiquity 3, 1984, 222 ("since Ovid composed with great speed, an unfinished Metamorphoses is likely to have suffered from more serious defects than an unfinished Aeneid").

${ }^{11}$ Cf. por ejemplo Cic., De or. 3.203 (ed. Mankin: tum correctio uel ante uel post quam dixeris uel cum aliquid a te ipso reicias) o, en el mismo Ovidio, Pont. 4.12.25-6 (ed. Richmond: saepe ego correxi sub te censore libellos, / saepe tibi admonitu facta litura meo est, [...]); no va en contra el testimonio de Probo en Gelio 13.21.4, a propósito de Virgilio (librum manu ipsius correctum legi); un segundo uso de incorrectum, mucho más neutro, puede observarse en Ps.-Acrón a Hor. Epist. 2.1.167 (ed. Keller: quod omnes poetae carmina sua scripta incorrecta dimittebant).

${ }^{12}$ R. J. Tarrant, P. Ovidi Nasonis Metamorphoses, Oxford 2004, VI y “Ovid”, 27, respectivamente; para una opinión contraria, próxima a las tesis de O. Zwierlein (Die Ovidund Vergil-Revision in tiberischer Zeit, I: Prolegomena, Berlín - Nueva York 1999, 9), cf. 
cliché literario más: la ausencia en su opus maius de una extrema, ultima o summa manus, es decir, la posible presencia esporádica de meros expedientes a la virgiliana (tibicines).

Ovidio insistió en el mismo asunto al postular la inclusión de un breve prefacio para la obra, cuya transcripción se halla inserta en los tres últimos dísticos de Trist. 1.7, versos 35-40. Tal prefacio había de aparecer seguramente extra ordinem paginarum -Marcial, IX praef.- según sugiere la instrucción ovidiana previa a la cita (v. 34: praeponendos) y como también sugiere en parte el propio metro de los versos propuestos ${ }^{13}$. El epigrama en cuestión, reiterativo y también provisto de abundantes reminiscencias filiales, habría podido llegar a insertarse -en opinión de Luck- en una "primera edición" de la obra, suponiendo que ésta llegase a producirse alguna vez con carácter formal ${ }^{14}$ : hos quoque sex uersus in primi [Heinsius, frente al prima de los códices $\left.{ }^{15}\right]$ fronte libelli, / si praeponendos esse putabis, habe: / 'orba parente suo quicumque uolumina tangis [cf. Trist. 3.14.14-6, en términos similares], / his saltem uestra detur in urbe locus. / quoque magis faueas, non sunt haec edita ab ipso, / sed quasi de domini funere rapta sui. / quidquid in his igitur uitii rude carmen habebit, / emendaturus, si licuisset, erat'. Aunque no por su contenido, la inserción guarda cierta similitud genérica con el falso proemio virgiliano a Eneida (Ille ego qui quondam...; cf. Vita Donati, cap. 42) eliminado por Vario según el testimonio del gramático Niso y que, de acuerdo con la hipótesis de Conte, Ovidio pudo llegar a conocer e imitar en varios lugares ${ }^{16}$.

La existencia de una "segunda edición" a cargo del autor, defendida a veces para el caso de las Geórgicas virgilianas (libro 4 sobre todo), suele descartarse con buen fundamento para Metamorfosis ${ }^{17}$, máxime si se acepta que los

K. Vanhaegendoren, "Ovid, Metamorphoses 1.258: textual criticism and Ovidian mockery", L'antiquité classique 74, 2005, 200 n. 4.

${ }^{13} \mathrm{Cf}$. C. Buongiovanni, "L'epigramma prefatorio da Marziale a Sidonio Apollinare", Voces 20, 2009, 52-3, a propósito de la decisiva aportación ovidiana a este género.

${ }^{14} \mathrm{Ap}$. J. Richmond, "Manuscript traditions and the transmission of Ovid's works", en B.W. Boyd, ed., Brill's companion to Ovid, Leiden - Boston - Colonia 2002, 444.

${ }^{15}$ Lectura defendida frente a Diggle ("Notes", 410-1), con el significado de distinguished, por H. White, "Notes on Ovid's Tristia", Habis 36, 2005, 210-1. De ser así, no estaría de más aducir paralelos adjetivales como los de Trist.1.1.8 y 110, y, asimismo, la ilustrada prima tabella de Marcial 14.186 (Vergilius in membranis), ya como posible tecnicismo librario; los dos argumentos de Diggle contra el testimonio de Trist. 2.545 (ueteris... libelli, referido a los tres libelli del Ars) carecen, en nuestra opinión, de peso refutatorio ("But a work in three books is not the same as a work in fifteen books, and it suits Ovid's apologetic purpose to pretend that the Ars is a book of no great substance”, más n. 22), máxime tratándose del afectivo diminutivo libellus (J. F. Gaertner, Ovid. Epistulae ex Ponto, book I, Oxford 2005, 97-8).

${ }^{16}$ Cf., últimamente, L. Mondin, "Ipotesi sopra il falso proemio dell'Eneide", CentoPagine 1, 2007, 67-8, B. Kayachev, "Ille ego qui quondam: genre, date, and authorship", Vergilius $57,2011,76-7$

${ }^{17}$ Magnus, “Ovids Metamorphosen?”, 239 (“Aber die Hypothese einer doppelten Recension, 
versos de Trist. 1.7.35-40, fueron concebidos como prefacio para una edición ya considerada o sancionada como definitiva por su autor (Trist. 1.7.12: quae mando qualiacumque legas; cf., por ejemplo, 3.14.51: qualemcumque igitur uenia dignare libellum) y que anulaba esbozos previos que no se consideraban propiamente editados, lo que también suprime en principio la existencia -en el texto transmitido o receptus- de duplices lectiones y variantes de autor de mayor o menor extensión y entidad ${ }^{18}$. Es difícil admitir que pudiera haber grandes diferencias textuales - a las que, sin duda, habría expuesto la inverosímil difusión de un borrador con variantes- entre los varios exempla vagamente aludidos por el autor: su propio ejemplar doméstico, custodiado en los añorados curua scrinia por parte de su esposa Fabia (Trist. 1.1.117- $\left.8^{19}\right)$, el ejemplar a disposición del destinatario de Trist. 1.7 o el que cabe suponer al alcance del propio Augusto a juzgar por Trist. 2.63-4.

Nuestros dos autores experimentaron una primera recepción opuesta en cierto modo. Virgilio desarrolló su labor al calor de la corte de Augusto, que es la que auspició su conservación misma a instancias del propio princeps

unhaltbar und unfruchtbar wie sie ist, mag Mutter Ge barmherzig versehlingen, wie sie einst ihre verzweifelnde Tochter Daphne versehlang"), Tarrant, P. Ovidi Nasonis Metamorphoses, XXXIV-XXXV; para el caso de la primera edición de Amores, en cinco libros, la segunda de Heroidas o la revisión, tras la muerte de Augusto, del primer libro de Fastos, cf. Richmond, "Manuscript traditions", 444, así como, sobre Trist. y Pont., 445 n. 9.

${ }_{18}$ Tarrant, P. Ovidi Nasonis Metamorphoses, XXXIV-XXXV; en torno, por ejemplo, a Met. 1.544-7, donde Tarrant atetiza 544-5 (apuntando en aparato duplicem quandam recensionem ipsius poeta agnoscunt plerique; de manera similar en 6.282 o en 7.146-5), cf., tras la propuesta de Blänsdorf ("Entstehung und Kontamination der Doppelfassung Ovid, Metam. I, 544-547a”, RhM123, 1980, 138-51, esp. 146-7: “Die Überlieferung weist also darauf, daß in der ältesten rekonstruierbaren Form des Textes die Tellusversion im Haupttext stand, die Peneusversion die Marginalvariante war"), el sólido trabajo de Murgia, "Ovid Met.1.544-547", partidario de mantener el pasaje (tras, por ejemplo, E. K. Rand, "The new critical edition of Ovid's Metamorphoses”, CPh 11, 1916, 49), apoyándose en Verg. Aen. 4.24-5; su contribución no es tenida en consideración por J. Cardigni, "Un caso de posible variante de autor en Ovidio: Metamorfosis 1.544-547", Circe 9, 2004, 87-99, cuyo título evoca una vieja hipótesis de K. Büchner ("Überlieferungsgeschichte der lateinischen Literatur des Altertums", en H. Hunger et al., Die Textüberlieferung der antiken Literatur und der Bibel, mit einem Vorwort von M. Bodmer, München 1998 [ $2^{\mathrm{a}}$ ed; $1^{\mathrm{a}}$ ed. 1975 (1961)], 403) retomada por Blänsdorf, "Entstehung”, 151 ("Hieraus läßt sich vermuten, daß Ovid im noch nicht endgültig überarbeiteten Manuskript die beiden unfertigen Versionen stehenließ. Die von den Freunden Ovids pietätvoll bewahrte Doppelfassung erreichte die spätantike Überlieferung, und sie stand [...] durchaus nicht allein. Lactantius Placidus konnte aufgrund der Einfügungszeichen die Marginalfassung für die Fassung letzter Hand halten, während die Schreiber karolingischer Zeit, die die textkritischen Symbole nicht mehr verstanden, die zwei Fassungen in der beschriebenen Weise kontaminierten"). Para el caso particular del fragmento Paris. Lat. 12246 (s. IX) y sus duplices lectiones (no de autor), cf. Tarrant, "Metamorphoses", 277, P. Ovidi Nasonis Metamorphoses, XXV.

${ }^{19}$ No vemos necesaria la hipótesis de O. Pecere (Roma antica e il testo: Scritture d'autore e composizione letteraria, Roma - Bari; ed. digital 2015; $1^{\mathrm{a}}$ ed. 2010, s. p.) según la cual "gli exempla superstiti delle Metamorfosi, verso cui Ovidio orienta l'amico incaricato di pubblicarle, dovevano comprendere anzitutto la sua copia privata”. 
(máxime en el caso de 'su' Eneida: Trist. 2.533: et tamen ille tuae felix Aeneidos auctor $^{20}$ ); por el contrario Ovidio, sin ostentar nunca la condición ejemplar de su modelo, perdió por varios motivos el favor de Augusto y sintió peligrar la integridad y -sobre todo- la difusión de su obra más extensa, para cuya preservación solicitó auxilio al poderoso y erudito amigo destinatario de Trist. 1.7, quizá coincidente con el de Trist. 3.14 (cf.1-2: Cultor et antistes doctorum sancte uirorum, / quid facis, ingenio semper amice meo?). Se ha conjeturado que en 1.7 pedía socorro a su amigo Bruto ${ }^{21}$, interlocutor bien atestiguado en los Pontica, o bien al provecto Higino ${ }^{22}$, supuestamente al frente aún de la biblioteca griega y latina del Templo de Apolo Palatino (praefuit Palatinae bibliothecae, según Suet., Gramm. et rhet. $20.2^{23}$ ), en la que no había copia de Met. a juzgar por Trist. 3.1.65-8 (quaerebam fratres, exceptis scilicet illis / quos suus optaret non genuisse pater: / quaerentem frustra custos me sedibus illis / praepositus sancto iussit abire loco), y, de ser así, posible garante de una óptima conservación de la obra ${ }^{24}$. Tanto Bruto como Higino han sido propuestos también como destinatarios de Trist. $3.14^{25}$. Zwierlein supuso que Julio Montano fue el des-

${ }^{20}$ Cf. B. Gibson, "Ovid on reading: reading Ovid. Reception in Ovid Tristia II", JRS 89, $1999,35$.

${ }^{21}$ Así A. L. Wheeler, Ovid in six volumes, VI: Tristia, Ex Ponto, $2^{\mathrm{a}}$ ed. (revisada por G.P. Goold), Cambridge (Mass.) - Londres, 1988 [1 $1^{\mathrm{a}}$ ed. 1924], 36 n. 3, Gaertner, Ovid, 93, quien opina que Bruto, destinatario también de Pont.1.1 (de finales del $13 \mathrm{~d}$. C.), "played a key role in the circulation of Ovid's exile poetry in Rome (not a publisher in the modern sense [...]"), como corrobarían Pont 3.9.51-6, 4.6.17-8.

${ }^{22}$ Así A. Grisart, "La publication des Métamorphoses: une source du récit d’Ovide (Tristes 1.7.11-40)", en Atti del convegno internazionale ovidiano - Sulmona 1958, Roma 1959, 153-5.

${ }^{23}$ Sobre el posible origen griego de la expresión cf. P. White, “Pomponius Macer' and Ovid”, $C Q 42,1992,213$ n. 9.

${ }^{24}$ Tarrant, "Ovid", 15, 30 n. 74. En opinión de R. A. Kaster (C. Suetonius Tranquillus. De grammaticis et rhetoribus, Oxford 1995 [reimpr. 2003], 212) la hipótesis de que fuera Higino carece de base firme y es improbable por razones cronológicas (Trist. 3 se concluye c. $10 \mathrm{~d}$. C., cuando Higino tendría unos 70 años; es dudoso que aún dirigiera la Biblioteca y que pudiera ejercer -quizá sin recursos, frente a lo que sugiere además 1.7.6-8- de valedor de nadie). La iniciativa pudo llegar a resultarle gravosa en lo personal: cf., tras van de Woestijne, A. Le Bœuffle, "Recherches sur Hygine", REL 43, 1965, 288-9, L. D. Bruce, "The procurator bibliothecarum at Rome", The Journal of Library History 18, 1983, 150 (“As a consequence of his relationship with Ovid, Hyginus may have lost imperial favor, for Suetonius relates that he died in complete poverty despite writings encompassing many subjects: agriculture, history, religion, a commentary on Vergil, and archaeology"), T. K. Dix, "Ovid strikes out: Tristia 3.1 and the first public libraries at Rome", The Augustan Age 7, 1988, 27-35. En todo caso, la desgracia de Higino pudo tener cualquier otro tipo de origen, según señaló Kaster, C. Suetonius Tranquillus, 213; por lo demás, Ovidio no menciona el nombre de Higino (familiarissimus según Suetonio) en ninguna de sus obras conservadas; no creemos tampoco que el sensu... amaro de Pont.1.2.37, sea, en vez de casual, alusión alguna a la erudición de Higino en materia virgiliana (cf. Georg. 2.247).

${ }^{25}$ Lo cree dirigido también a Higino, después de Luck, E. Baeza Angulo, Publio Ovidio 
tinatario de ambos poemas y, por tanto, el "editor" de Ovidio ${ }^{26}$, admitiendo el bien atestiguado pero ambiguo conficis de Trist.3.14.5 (cf. ed. Hall: suscipis $[. .$.$] mea carmina [. .$.$] ?) como un tecnicismo editorial.$

Fuera cual fuera el efecto de la prohibición de Augusto ${ }^{27}$, tanto el "escolar" y difundidísimo Virgilio como el relegado Ovidio (al menos en lo referente a Metamorfosis) podían adquirirse en el Foro a finales del siglo I d. C., según demuestra Marcial en sus Apophoreta 14.186 (ed. Shackleton Bailey: Vergilius in membranis: Quam brevis immensum cepit membrana Maronem! / ipsius vultus prima tabella gerit) y 192 (Ovidi Metamorphosis in membranis: Haec tibi, multiplici quae structa est massa tabella, I carmina Nasonis quinque decemque gerit). Ambos códices se hallarían incluidos entre los regalos baratos (sortes pauperis) a disposición del comprador, lo que, de ser así y dado que constarían de varios cientos de folios cada uno, apenas resulta comprensible ni siquiera como ironía literaria por parte de Marcial ${ }^{28}$. Conviene destacar que el corpus virgiliano consagrado como tal todavía podría caber en su integridad en un solo códice, como podría ocurrir también en el caso de Metamorfosis, a juzgar por lo que ya sabemos sobre este tipo de manufacturas manuscritas antiguas y tardoantiguas (tanto paganas como cristianas), si bien no puede excluirse - pese a lo que sugiere el structa massa del poema $192^{29}$ - que Marcial aluda de manera genérica a un lote de, por ejemplo, tres códices de menor extensión, de cinco libros cada uno. Cosa distinta ocurría con la extensa y variada producción ovidiana en su totalidad, de muy difícil transmisión conjunta y cuyas obras, de hecho, ofrecen tradiciones manuscritas bastante heterogéneas.

Poco puede llegar a saberse de las primeras fases de transmisión manuscrita de Eneida y Metamorfosis desde un punto de vista material, pero sí cabe observar una serie de rasgos objetivos que diferencian plenamente sus tradiciones textuales más remotas y que pasamos a enumerar brevemente:

Nasón. Tristezas, Madrid 2005, 111 n. 125; se dedicaría a "a poet-friend of greater age than Ovid" según Wheeler, Ovid, XV; a Bruto en opinión de Gaertner, Ovid, 93 y 158.

${ }^{26}$ Zwierlein, Die Ovid- und Vergil-Revision, 5 n. 2.

${ }^{27}$ Una mera "interrupción” en la transmisión de Met., según J. G. Clark en su "Introduction" a J. G. Clark - F. T. Coulson - K. L. McKinley, eds., Ovid in the Middle Ages, Cambridge 2011, en 2 , quien añade (3): "There can be no doubt that a certain notoriety, a danger even, surrounded the name of Ovid in the decades following his death. With the conceit characteristic of a following generation, the stylists of the post-Augustan age tempered their evident admiration with a tone of mild reproach".

${ }^{28}$ Cf. T. J. Leary, Martial. Book XIV: the Apophoreta, Londres 1996 (reimpr. 2002), 19-21, y, en referencia a un posible exposé humoristique por parte de Marcial, É. Prioux, "Le fromage et le dentifrice: le couple Virgil - Ovide dans les Xenia et les Apophoreta de Martial", en S. Clément-Tarantino - F. Klein (dir.), La représentation du 'couple'Virgile - Ovide dans la tradition culturelle de l'Antiquité à nos jours, Villeneuve d'Asqc 2015, 120-1.

${ }^{29}$ Cf., por ejemplo, Met. 1.70 [cód. M et al.], en combinación con Met. 1.677: structis cantat auenis. 
-frente a lo que ocurre con Virgilio, es total la ausencia de papiros ovidianos conservados ${ }^{30}$;

-es prácticamente completa $-\mathrm{y}$ esencial en lo que al texto se refiere- la transmisión tardoantigua que se conserva de Virgilio, frente a lo que ocurre en el caso de Ovidio, con sólo un magro resto: el manuscrito G de Pontica (Guelf. Aug. $4^{\mathrm{O}} 13.11=$ CLA IX 1377), consistente en un par de pequeños fragmentos en uncial del segundo cuarto del siglo V (Pont. 4.9, 101-8 y 127$33 ; 12,15-9$ y $41-4)$, procedentes quizá de Bobbio y reutilizados a principios del siglo VIII, en el entorno de Luxeuil, como palimpsesto, con Agustín de Hipona, Epist. y Serm., como scriptio superior ${ }^{31}$;

-la transmisión ovidiana, a diferencia de la virgiliana o la horaciana, carece de ejemplares completos que puedan datarse en los siglos IX, X o primera mitad del $\mathrm{XI}^{32}$, si bien quedan fragmentos de importancia que prueban - si era necesario- su existencia;

-ausencia de una labor crítica documentada en torno a Ovidio, en contraste con la que la tradición indirecta virgiliana atribuye a Probo y otros gramáticos o eruditos ${ }^{33}$; las abundantes noticias, espurias o no, referentes a la difusión primitiva de Virgilio brillan por su ausencia en la de Ovidio, carente de disturbadores "autógrafos" en circulación y aparentemente libre del asedio sistemático de los obtrectatores (quienes se ensañaban sobre todo con los autores de primer rango indiscutible $\left.{ }^{34}\right)$;

-la tradición antigua de escolios, tan rica en lo referente al corpus virgiliano, es casi inexistente en el caso de Ovidio, autor que apenas se aduce incluso, como autoridad, en los comentarios de su rival literario ${ }^{35}$; solamente los esco-

${ }^{30}$ Richmond, "Manuscript traditions", 446 ("No papyrus of Ovid survives [...]”), Clark, "Introduction", 4 ("Certainly there is little in the evidence of papyri to indicate an unusual intensity in transmission at least to the outer reaches of the empire").

${ }^{31}$ Richmond, "Manuscript traditions", 446-7; puede consultarse una excelente digitalización del códice y su descripción en la página correspondiente de la Herzog August Bibliothek de Wolfenbüttel (< http://diglib.hab.de/mss/13-11-aug-4f/start.htm >); según Gaertner, Ovid, 40 n. 174 (tras Richmond), el códice "is not the archetype of the remaining tradition". Por lo demás, convendrá recordar que ni siquiera un pecio tan minúsculo subsiste, por ejemplo, de la transmisión tardoantigua de Horacio.

${ }^{32}$ Sobre el posible refugio periférico del corpus (Irlanda, Península Ibérica o Norte de África) en épocas anteriores, cf. Rand, "The new critical edition", 48, 55, Clark, "Introduction", 6.

${ }^{33}$ En el caso de Montano, frente a la hipótesis de Zwierlein, Die Ovid- und Vergil-Revision, quien le atribuye "raffinierte Fälschungen" en todo el corpus ovidiano (5), cf. también, tras Zetzel y Galinsky, Tarrant, P. Ovidi Nasonis Metamorphoses, XXXIV n. 47. La enorme diferencia entre las muchas citas virgilianas presentes en los gramáticos y el magro número que éstos ofrecen de Ovidio también ha sido destacada por P.L. Gatti, Ovid in Antike und Mittelalter. Geschichte der philologischen Rezeption, Stuttgart 2014.

${ }^{34}$ Como era el caso de Virgilio y Cicerón: X. Ballester, Los mejores títulos y los peores versos de la literatura latina, Barcelona 1998, 105.

${ }^{35}$ P.E. Knox, "Commenting on Ovid", en P.E. Knox, ed., A companion to Ovid. Blackwell companions to the ancient world, Chichester - Malden, 2009, 328 ("[...] Ovid, for whom no trace of an ancient commentary survives"), J. Haynes, "Citations of Ovid in Virgil's ancient 
lios de algunos manuscritos medievales de $I b i s$ remiten a erudición antigua ${ }^{36}$, así como los tituli y argumenta en prosa de Met. (narrationes) atribuidos desde el siglo XV a Lactantius (Lactancio Plácido, en principio ${ }^{37}$ ), escritos en los siglos IV-V ${ }^{38}$, recogidos quizá en un códice de los siglos $\mathrm{V}-\mathrm{VI}^{39}$ y transmitidos sobre todo en tres manuscritos de origen itálico (M, N y U).

No es posible saber lo que ha podido representar el mero azar en la conservación de materiales codicológicos, dentro de cada uno de los apartados antes mencionados, pero todos los datos objetivos se complementan de manera armónica y, entre lo cuantitativo y lo cualitativo, apuntan en una misma dirección: una difusión mucho más restringida del corpus ovidiano y menor actividad escolar y erudita en torno al mismo, tanto en sus primeras fases como -en lógica consecuencia- durante el primer Medievo.

\section{LA CUESTIÓN DEL ARQUETIPO}

Lo referente al arquetipo - a menudo sin solución, pero ineludible en cualquier historia del texto ${ }^{40}$ - ofrece también ciertos rasgos comunes y ciertos rasgos distintivos en las dos transmisiones que nos hemos propuesto confrontar.

commentators”, en Ch. S. Kraus - Ch. Stray, eds., Classical commentaries: explorations in a scholarly genre, Oxford 2016, 216-32.

${ }^{36}$ R. J. Tarrant, "Ibis", en L.D. Reynolds, ed., Texts and transmission. A survey of the Latin classics, repr. with corr., Oxford 1986 [1983], 274; esta obra ha recibido particular atención en la monografía de Gatti antes mencionada (Ovid in Antike und Mittelalter).

${ }^{37}$ Cf. B. Otis, "The argumenta of the so-called Lactantius", HSCPh 47, 1936, 131-63, R. Tarrant, "The narrationes of 'Lactantius' and the transmission of Ovid's Metamorphoses", en O. Pecere - M. D. Reeve, eds., Formative stages of classical tradition: Latin texts from Antiquity to the Renaissance, Spoleto 1995, 83-115, Richmond, "Manuscript traditions", 445 y 469, B. Munk Olsen, L'étude des auteurs classiques latins aux XI et XII e siècles, tome IV - $1^{\text {re }}$ partie: La réception de la littérature classique. Travaux philologiques, París 2009, 92.

${ }^{38}$ Tarrant, P. Ovidi Nasonis Metamorphoses, XXVI.

${ }^{39}$ En capital rústica y con los argumenta (y quizá escolios procedentes de comentarios) al margen, según Otis, "The argumenta", 160; sobre el carácter escolar y, en buena parte, progimnástico del material, cf. R. J. Hexter, "Medieval articulations of Ovid's Metamorphoses: from Lactantian segmentation to Arnulfian allegory", Mediaevalia 13, 1987, 67.

${ }^{40}$ Subscribimos las afirmaciones de F. Dolveck en su reseña a R.J. Tarrant, Texts, editors, and readers: methods and problems in Latin textual criticism. Roman literature and its contexts, Cambridge 2016 (< http://bmcr.brynmawr.edu/2016/2016-11-46.html >): "the first part of the editorial work, that is the reconstruction of the archetype, has nothing to do with rhetorical play. It is a matter of pure logic, and as such is indeed the most 'scientific' part of the Humanities. Except in a few cases, stemmatics is a legitimate construction from the available evidence". Un estema no impone nada como fuerza ciega; en la estemática no se "cree", ni contar con su recurso "hace más feliz" al filólogo (como cándidamente sugería L. Canfora, "Un modesto sucedáneo: el arquetipo", en El copista como autor [= Il copista come autore, Palermo 2002, tr. R. Bonilla Cerezo], Madrid 2014, 32); negar su validez en abstracto es como renegar, en Física, de la ley de la gravedad: un error, una superstición y, a menudo, una inelegante desconsideración hacia la nada desdeñable Filología que nos ha precedido. 
Como es sabido, el procedimiento de las faltas comunes apenas permite la reconstrucción de arquetipo alguno en el caso de Eneida, al igual que ocurre con las demás obras del corpus virgiliano; en la senda de Pasquali, se ha propuesto su identificación con la propia edición de Vario, a la que remontarían recta uia los códices antiquiores, pero ha habido otras propuestas de cronología diversa, postulándose ejemplares de los siglos II, III y IV, de modo que puede seguirse afirmando que la historia más primitiva del texto virgiliano todavía debe perfilarse en lo esencial ${ }^{41}$.

Los errores coniunctivi comunes a toda la tradición manuscrita tampoco aportan indicios decisivos en el caso de Metamorfosis, aunque - haciendo caso omiso de las muchas interpolaciones supuestas, siempre de valor estemático en principio- ha solido repararse en el ejemplo de 1.580 (Apidanosque / Eridanosque; cf. Verg. Georg. 4.372), error repetido en 7.228 (salvo en Planudes ${ }^{42}$ ), así como en lugares de aparente sutura que podrían estar reflejando, en principio, el deterioro de una fuente común (así en 1.91-3 o en 304-53). Tras el trabajo pionero de Magnus, ampliamente criticado a este respecto por Rand y sus discípulos, o, muchos años más tarde, del ambicioso estudio de Luck (quien, en alarde lachmanniano, llegó a concretar la hipótesis de un arquetipo tardoantiguo, en tres códices de 150, 156 y 160 folios respectivamente y de 13 líneas por página ${ }^{44}$ ), la contribución de referencia es hoy la de Tarrant, pese a los dudosos criterios "estemáticos" — o más bien sólo "taxonómicos"aplicados a veces por este editor, como el de reunir códices bajo una misma sigla en función de su cronología y no de su configuración textual ${ }^{45}$, entre otros. En lo esencial, Tarrant asumió las dos familias sólidamente establecidas

${ }^{41}$ En general cf. Á. Escobar, "La tradición antigua del texto virgiliano: notas acerca de algunas hipótesis recientes”, ExClass 12, 2008, 25-47.

42 Richmond, "Manuscript traditions", 443 n. 3.

${ }^{43}$ Rand, "The new critical edition", 53, R. T. Bruère, "The manuscript tradition of Ovid's Metamorphoses", HSPh 50, 1939, 99, Büchner, "Überlieferungsgeschichte”, 403 (con referencia a 1.70, 5.541, 8.186 y 9.347, siguiendo a G. Pasquali, Storia della tradizione e critica del testo. Seconda edizione con nuova prefazione e aggiunta di tre appendici, Florencia 1962 [1 $1^{\underline{a}}$ ed. 1952], 388-9); desde el punto de vista textual cabe comparar el problema que subyace en Georg. 4.290-3 o en Aen. 10.661-5, 714-8.

${ }^{44}$ Las especulaciones de G. Luck (Untersuchungen zur Textgeschichte Ovids, Heidelberg 1969) obtuvieron respuesta bastante crítica de S. Borzsák, Gymnasium 77, 1970, 332-4, M. Winterbottom, “The text of Ovid”, CR 21, 1971, 208-9, o H. Dörrie, Gnomon 46, 1974, 664-9. Añadiríamos que la supuesta impaginación de tal modelo, coincidente con la del Veronensis XL (38) de Virgilio (escrito a finales del s. V, quizá en el norte de Italia), entraña en principio muy amplios márgenes, propios de un ejemplar de lujo o destinado a la incorporación de notas marginales.

45 Tarrant, $P$. Ovidi Nasonis Metamorphoses, XXXVI; cf. P. Fedeli, res. de Tarrant, $P$. Ovidi Nasonis Metamorphoses, Gnomon 79, 2007, 607, G. Luck, res. de Tarrant, P. Ovidi Nasonis Metamorphoses, ExClass 9, 2005, 249-71, esp. 251-2, 270; el estema reeditado por Tarrant en Texts, editors, and readers, 54, con correcciones respecto al de 2004, no modifica sustancialmente su propuesta; en p. 48 reitera la imposibilidad de establecer un estema (cf. ya $P$. Ovidi Nasonis Metamorphoses, VII) y la necesidad de un procedimiento ecléctico. 
por Magnus, pero renunció a intentar remontarse más allá de esa constatación: "codices [...] antiquiores in duas classes uel familias diuiduntur ${ }^{46}$ [...] Sed noli, quaeso, sperare lectiones archetypi cuiusdam omnium codicum hoc stemmate posse restitui [...]", de modo que, según concluía, "ipsae lectiones sint ponderandae, non codices" ( $\mathrm{p}$. XXVII). Claramente decantado 'de la parte del texto' -dicho sea parafraseando a Cavallo y su emblemático volumen Dalla parte del libro- Tarrant desestimaba la posibilidad de establecer un arquetipo y de postular su cronología, ya fuera antigua, tardoantigua o carolingia ${ }^{47}$.

Hoy sólo cabe especular sobre la posibilidad de que el recurso a un mayor número de ejemplares de Metamorfosis en mayúscula o en minúscula primitiva, por desgracia no conservados, hubiera facilitado la investigación del problema, pero también es muy posible que tales testimonios nos condujesen, sencillamente, a una reconstrucción muy próxima mutatis mutandis a la que parece observarse en Eneida: a un arquetipo antiguo cuya realidad histórica sólo cabe suponer a partir de noticias de valor muy desigual (como son las anteriormente señaladas en los Tristia, provenientes del propio autor), quizá provisto de variantes ${ }^{48}$, y a hiparquetipos de enorme indefinición, como es el caso de los tres fragmentos del siglo IX así considerados por Tarrant, todos ellos de muy escasa extensión y que se hacen derivar directamente del arquetipo al igual que un supuesto testimonio $(\mathrm{x})$, en lo que, de lo contrario, sería un estema bífido (familias $\Delta$ y $\Sigma$ en la representación de Tarrant) del tipo más convencional ${ }^{49}$.

${ }^{46} P$. Ovidi Nasonis Metamorphoses, XX, en referencia a códices 'lactancianos' (familia $\Delta$ de Tarrant) y 'no lactancianos' $(\Sigma)$, a veces denominados 'vulgata'; este término se ha aplicado en ocasiones a la familia X de Magnus, así como a un supuesto texto común de los siglos XIIXIII no portador del material lactanciano; estaría representada por ejemplo en el Marc. 223 (F en Tarrant), provisto con el final del libro 14 y el libro 15, ausentes en O (Rand, "The new critical edition", 48); Tarrant destacó la importancia al respecto del Vat. Pal. 1669, hermano del Sangal. 866 ("Metamorphoses”, en Reynolds, Texts, 281, P. Ovidi Nasonis Metamorphoses, $\mathrm{XXIV);} \mathrm{a} \mathrm{las} \mathrm{interrelaciones} \mathrm{o} \mathrm{coincidencias} \mathrm{entre} \mathrm{ambos} \mathrm{hiparquetipos} \mathrm{(O} \mathrm{y} \mathrm{X} \mathrm{en} \mathrm{la} \mathrm{edición} \mathrm{de}$ Magnus) ya aludió ampliamente Rand, "The new critical edition", 53-5 (cf. 55: "Such evidence leads us to associate these two groups still more closely, and not to regard one as an ancient scholarly recension and the other as an ancient vulgate"). Tarrant admite y describe asimismo otras relaciones estemáticas suficientemente claras.

${ }^{47}$ Tras las investigaciones de Rand, Smith o Bruère en la misma dirección, cf. W. S. Anderson, P. Ovidius Naso. Metamorphoses, ed. ster. ed alt. [1982], Berlín - Nueva York 2008, V-VI ("ratio simplicissima et inde gratissima iamdiu fuit haec: unum codicem solum ad aetatem Caroli Magni superstitem exemplar fuisse omnium codicum posteriorum [...] eis assentior qui affirmant unum solum exemplar satis verisimile esse. nam adhuc non redargutum est. non solum complures errores, qui omnes libros deformant, sed etiam quaedam lectiones quae dicuntur duplices ante aetatem Caroli regis et ideo ante omnes codices quos nunc habemus ortae sunt").

${ }^{48}$ Como el imaginado por Ribbeck para el caso de Virgilio ("unum archetypum [...] oppletum variis lectionibus et glossematis", ap. M. Geymonat, "Eneide: La problematica ecdotica del testo”, en F. della Corte, Enciclopedia Virgiliana, II (DE-IN), Roma 1985, 292); para el caso de Met. cf. todavía Pasquali, Storia, 389, Büchner, "Überlieferungsgeschichte", 403-4.

${ }^{49}$ Cuatro hiparquetipos o ediciones antiguas postulaba Pasquali, Storia, 387-90, seguido 


\section{LA TRANSMISIÓN MEDIEVAL}

Al problema del arquetipo se une en el caso de Ovidio el de una transmisión de testimonios muy abundantes, próximos al medio millar ${ }^{50}$, pero muy tardíos en comparación con Virgilio, ya que los primeros antiquiores completos de Metamorfosis datan de finales del XI o principios del XII. Ésta se caracteriza, además, por la contaminación, considerada muy intensa en varias ramas (si bien a partir de un mismo foco [x] según Tarrant, $\mathrm{X}$ en la versión corregida de su estema). Se trata, por tanto, de una transmisión horizontal todavía por analizar en su conjunto, pese a esporádicos avances como el alcanzado en el estudio de N, Neap. Bibl. Nat. IV.F.3, importante testigo de los siglos XI-XII contaminado un siglo más tarde, o en el de U, Urb. Lat. 341. Aunque ha llegado a afirmarse que un estema puede resultar inútil en el caso de Ovidio ${ }^{51}$, nosotros consideramos que sería un instrumento filológico de gran valor (particularmente para el estudio de la contaminación misma, cuyas vías no son discernibles sin su recurso) y que podría ayudar a entender y valorar por ejemplo, desde criterios filológicos y no de mero gusto, un buen número de lecturas de carácter excéntrico ${ }^{52}$.

por Büchner, "Überlieferungsgeschichte", 403. Sobre el arquetipo de Trist. cf. Hall, $P$. Ovidi Nasonis Tristia, XII; el error de Trist. 1.11.12, mens releuata (codd.), donde la lectura supuestamente correcta, cura leuata, se restituye sólo gracias a CIL VI $9632=C L E$ 89, 1-2 (I-II d. C.), parece considerarlo Hall anterior a la propia constitución del arquetipo; pese a la vehemencia del tonante Housman al respecto, J. Trappes-Lomax se atrevió a considerar mens releuata como "unsatisfactory but not quite intolerable" "Ovid Tristia 2.549: how many books of Fasti did Ovid write?”, CQ 56, 2006, 633), quizá por no descartar la remota posibilidad de una excelente conjetura antigua (cf. Quint. 9.3.44: prioris sententiae uerbum ultimum ac sequentis primum frequenter est idem, quo quidem schemate utuntur poetae saepius, y, con referencia a este vicio del estilo ovidiano, Conte ad Aen. 10.705, Ope ingenii: experiences of textual criticism, Berlín-Boston 2016, 77). Sobre el arquetipo de los siglos IV-V de Pont. cf. R. J. Tarrant, "Epistulae ex Ponto", en Reynolds, Texts, 263, J.F. Gaertner, "Zum Text und zur Textgeschichte der Epistulae ex Ponto (zu Ov. Pont. 1,1,41-44; 1,6,45-46; 4,9,130-132)", RhM 147, 2004, 63-71, esp. 63-4.

${ }^{50}$ Richmond, "Manuscript traditions", 449; se trata de la mitad de códices, más o menos, que en el caso de Virgilio y el número se halla también muy por debajo del de un Horacio o un Terencio; una descripción sumaria en el utilísimo repertorio publicado por el equipo de Ramírez de Verger en < http://www.uhu.es/proyectovidio/esp/20_manuscritos.html >.

${ }^{51}$ Así Knox, res. de Hall, P. Ovidi Nasonis Tristia: "In the case of the Tristia, as in Ovid's other works, construction of a stemma, even if it were possible, would avail the editor nothing"; un juicio sumarísimo en la misma línea ha emitido más recientemente K. S. Myers, Ovid. Metamorphoses. Book XIV, Cambridge 2009, 23 ("No pure strain of the textual tradition can be identified or given preferential treatment"), sintiéndose así autorizada a ofrecer un singular aparato con sólo dos siglas, saludado por J. B. Solodow en < http://bmcr.brynmawr. edu/2011/2011-03-55.html > ("In regard to the (very slim) apparatus criticus, Myers has adopted a scheme of drastic economy: M represents the entire manuscript tradition, $m$ a part of it. Yet in a tradition where there are no decisively preponderant manuscripts and where much horizontal contamination is found, as a result of which texts are invariably eclectic, this makes a good deal of sense").

${ }^{52}$ Así en Met. 6.58 (pauiunt, frente al trivial feriunt; cf. Sen. Epist. 90.20, M. Possanza, 
El estema de Tarrant se limita a incluir los manuscritos más antiguos y, en principio, relevantes. La inclusión de códices más recientes se considera muy difícil - al menos desde los procedimientos más tradicionales de la crítica ${ }^{53}$ a causa sobre todo de la contaminación señalada, que se intensifica a partir del siglo XII en paralelo a la recuperación de Ovidio como autor canónico. Es muy escasa todavía la identificación de manuscritos procedentes de entornos claramente escolares ${ }^{54} \mathrm{y}$, como en el caso de la tradición virgiliana, es mucho lo que queda por investigar asimismo sobre historia de los manuscritos, por ejemplo en materia de poseedores y de prosopografía ${ }^{55}$.

En ausencia de una tradición conservada tan antigua como la virgiliana, con absoluta predominancia del testimonio de tres códices (MPR) e, incluso, de un codex optimus más o menos reconocido $(\mathrm{M})$, la ordenación y selección de testigos es mucho más difícil en el caso de Metamorfosis, ya que ni siquiera su códice M (el relevante Marc. 225 de la segunda mitad del siglo XI tan apreciado en su día por Heinsius o por Merkel) ha seguido considerándose como optimus ${ }^{56}$. Es comprensible, por tanto, que Tarrant retomase la defensa cerrada del eclecticismo ecdótico (p. XXXII: "apparet textum

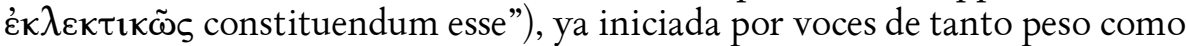
la de Goodyear ${ }^{57}$, aludiendo a la dificultad de preferir una familia frente a

"Editing Ovid: immortal works and material texts", en Knox, A companion, 316-7) o en 10.637 (quod facit G, frente al 'conservador' quid facit; sobre el valor de G, Sang. 866, en general, cf. S. Heyworth, "The OCT Metamorphoses", CR 57, 2007, 104-5).

53 Tarrant, P. Ovidi Nasonis Metamorphoses, VII; es decir, de un análisis todavía al margen de metodos que aún deben adoptarse en nuestra Filología como el de 'coherencia genealógica', aplicados a otras transmisiones ampliamente contaminadas y tan diversas como la neotestamentaria o la del Avesta (cf. por ejemplo, respectivamente, G. Mink, "The coherencebased genealogical method - What is it about?", 2002 (< https://www.uni-muenster.de/ INTF/Genealogical_method.html $>$ ), A. Cantera, "The problems of the transmission of the Avestan texts and the tools for Avestan text criticism (TATEC)", en T. Andrews - C. Macé, eds., Analysis of ancient and medieval texts and manuscripts: digital approaches, Lectio 1, 2015, 95-116).

${ }^{54}$ En general cf. Richmond, "Manuscript traditions”, 445, 452 n. 38, así como Clark, "Introduction", con referencias de interés a la implantación ovidiana en las escuelas desde comienzos del siglo IV, pese a reticencias o prevenciones esporádicas (de apologetas como Lactancio a Conrado de Hirsau o Guiberto de Nogent). Sobre el Dertus. 134 (siglos XII-XIII), por ejemplo, cf. M. Librán Moreno, "Colación de Dertusensis 134 (Ov. Metamorphoseon libri XV)", ExClass 10, 2006, 84.

${ }^{55}$ Un caso excepcional es el del Thomas iudex corrector del Vat. Lat. 5859, estudiado por A. Manfredi ("Un altro codice per Zanobi da Strada”, en A. Manfredi - C. M. Monti, eds., Le antiche et le moderne carte. Miscellanea di studi in memoria di Giuseppe Billanovich, Padua - Roma 2007, 361-95).

${ }^{56}$ Cf. Rand, "The new critical edition", 60, Otis, "The argumenta", 141, 162 ("not the most excellent but the most doctored Ovidian text"), Diggle, "Notes", 413 ("M is prone to idiosyncratic blunders").

${ }^{57}$ Res. de A. S. Hollis, Ovid, Metamorphoses, book VIII, Oxford 1970, en JRS 61, 1971, 306 ("For the present, and probably the future, eclecticism, which is much the same as a return to Heinsius, is the only sane approach to the editing of the Metamorphoses [...]"); cabe comparar 
otra o un códice frente a otros ( $i b .:$ "Neutra enim codicum classis tanta pollet auctoritate ut eius lectiones constanter praeferri mereantur, nedum codices singuli”), así como, de hecho, invitando en última instancia a poner todas las variantes existentes en la tradición al mismo nivel y como posibles opciones directas con plena validez (en vez de, por ejemplo, como verosímiles conjeturas tempranas $\left.{ }^{58}\right)$. Ignoramos si, en términos absolutos, el número de variantes que ofrece el texto de Metamorfosis es mayor que el que ofrece el de Eneida, como invita a pensar el mero cotejo de ediciones al uso y, más aún, el de aparatos críticos de cierta extensión ${ }^{59}$, pero, tanto en un caso como en el contrario, su escrutinio a ciegas sería demasiado arduo y sólo podemos, en fin, suscribir las poco 'populares' palabras de S.P. Oakley a propósito de una muy reciente monografía de Tarrant ${ }^{60}$ : "[...] am accordingly suspicious of a popular doctrine: that in these and some other traditions any MS. may potentially contain inherited truth not found elsewhere". Dicho de otro modo: eliminatio lectionum singularium, desde la certeza de que una variante aislada en el seno de una tradición apenas puede remontar, en principio, a un texto auténtico, como bien sabe cualquiera que haya afrontado la elaboración del estema de una tradición textual normalmente constituida.

\section{CONCLUSIONES: DOS FISONOMÍAS TEXTUALES CONFRONTADAS, UN MISMO PRO-} CEDER ECDÓTICO

Es evidente que las fisonomías de las dos tradiciones analizadas durante este breve ejercicio de "comparatismo" histórico-textual, reflejadas en los rasgos generales que hemos procurado señalar así como en sus propios errores más frecuentes o característicos, presentan rasgos diferenciales de cierta relevancia y bien configurados desde antiguo. Es verosímil que ambas tradiciones fueran confluyendo poco a poco por el hecho de transitar ámbitos y vías de

Tarrant, "Metamorphoses", 277 ("enlightened eclecticism based on sense and usage must guide future editors just as it did the greatest of their predecessors, Nicolaus Heinsius").

${ }^{58}$ La misma opinión en Richmond, "Manuscript traditions", 470-1 y 472 (“correct readings may be found in isolated places"). Tarrant renunciaba incluso a la posibilidad de acordar un criterio ortográfico consistente: R. J. Tarrant, "Editing Ovid's Metamorphoses: problems and possibilities" [res. de Anderson, P. Ovidius Naso. Metamorphoses], CPh 77, 1982, 348, P. Ovidi Nasonis Metamorphoses, XXXVII ("Fateor me quaedam magis arbitrio quam consilio statuisse"); pese a señalar la conveniencia de adoptar algunas convenciones, tampoco parece haber sido un editor del todo coherente al respecto (cf., por ejemplo Luck, res. de Tarrant, $P$. Ovidi Nasonis Metamorphoses, 270).

${ }^{59}$ Como el elaborado por P. Fàbregas Salis, Edición crítica y comentario textual del libro $X$ de las Metamorfosis de Ovidio, Tesis Doctoral (dirs. J. L. Vidal - A. Ramírez de Verger), Universitat de Barcelona, 2016.

${ }^{60}$ Res. de Tarrant, Texts, editors, and readers, en JRS 106, 2016, 361. Cf., por el contrario, A. Ramírez de Verger en su correspondiente reseña (Ecdotica 13, 2016, 215-26), en 218: “Acierta una vez más T. en que en las Metamorfosis de Ovidio, como en las transmisiones abiertas, «every lectio recentior is potentially a lectio vetustior for which an older witness happens not to survive»". 
transmisión comunes, hasta acabar contaminándose mutuamente, sobre todo desde época altomedieval y en la dirección que iba de Virgilio - modelo de modelos- a Ovidio (circunstancia muy patente en alternancias que recurren en las dos transmisiones, del tipo corpus / pectus, pinus / tinus, etc.). Cabe especular asimismo sobre los motivos que pudieron llegar a hacer del retórico Ovidio - frente al alusivo y elusivo Virgilio o el marmóreo Horacio- un autor mucho más expuesto a la deturpación en todo momento: el estilo barroco y calimaqueo o juguetón que exhibe el texto del de Sulmona ${ }^{61}$ había de mostrar su pronto reflejo en determinadas prácticas de copia, sobre todo en los entornos eruditos y escolares, exacerbando todo tipo de aemulatio. Esta posibilidad se ve refrendada, en nuestra opinión, por la gran cantidad de variantes "metonímicas" (miméticas, pertenecientes al eje de la combinación más que al del paradigma ${ }^{62}$ ) que el texto ovidiano ofrece en comparación con el de Virgilio, así como de glosas y demás variantes aparentes, de carácter no mecánico o involuntario. No es casual, desde este mismo punto de vista, que el problema de los versos interpolados sea característico de la transmisión ovidiana, sobre todo en el caso de Metamorfosis ${ }^{63}$, por mucho que el proceder ecdótico seguido al respecto haya sido a veces dudosamente objetivo y sobre todo, en bastantes ocasiones, de muy escasa consistencia (buen ejemplo puede ser quizá el de Met.7.135-6 o el de 10.347-8: melius abessent en ambos casos, según el "probiano" dictamen de Tarrant). No es menester ver en los artífices de la transmisión ovidiana, por ello, más falsarios de lo necesario, ya que la intervención de los lectores ha respondido también en este caso -como en el de Virgilio, según defendió Timpanaro hasta el final- a los mecanismos más habituales de la transmisión antigua, tardoantigua y medieval ${ }^{64}$. En todo caso, conviene señalar que las consecuencias estemáticas de las interpolaciones ovidianas parecen haber recibido menor atención que en el caso de Virgilio, gracias por ejemplo a Courtney y a sus hipótesis - fundadas o no- en

${ }^{61}$ Murgia, “Ovid Met. 1.544-547”, 234-5.

${ }^{62}$ Á. Escobar, "La práctica de la collatio en la edición romana antigua", Actas del VIII Congreso Español de Estudios Clásicos (Madrid, 23-28 de septiembre de 1991), II, Madrid 1994, 603-10.

${ }^{63}$ Cf. R. J. Tarrant, “Toward a typology of interpolation in Latin poetry”, TAPhA117,1987, 281-98, P. Ovidi Nasonis Metamorphoses, XXXIII-XXXIV; Tarrant llega a atetizar la mitad aproximadamente del número de versos que condenó Merkel (unos 250 en Met.; cf. Luck, res. de Tarrant, 255, M. Possanza, res de Tarrant en < http://bmcr.brynmawr.edu/2005/200506-27.html >, Heyworth, "The OCT Metamorphoses", 105, G. B. Conte, Ope ingenii, 46-54), no siempre versos sueltos o pares de versos aislados (cf. por ejemplo 8.597-600b ó 603-8).

${ }^{64}$ Cf. Dolveck, res. de Tarrant, Texts, editors, and readers: "The vast majority of medieval scribes and scholars did exactly what we do: try to restore the text in front of them. Maybe they were wrong, maybe some even thought that they could challenge the greatest poets and prose-writers of ancient Rome, but they did not intend to deceive us and do not deserve our contempt"; en términos afines, Tarrant, P. Ovidi Nasonis Metamorphoses, XXXIII, Richmond, "Manuscript traditions", 449, Conte, Ope ingenii, 46 ("Neither do I believe that they were all motivated by the malice of the forger"). 
torno al supuesto arquetipo tardoantiguo del corpus virgiliano.

Tanto el texto de Eneida como el de Metamorfosis se hallan sólidamente fijados, debido a la alta calidad de conjunto de sus respectivas parádosis. Los criterios ecdóticos aplicados a una y otra obra apenas han diferido en esencia, pese a la gran diversidad que ofrecen las dificultades textuales más frecuentes en cada caso (arcaísmos y cuestiones de tradición indirecta en Aen., por ejemplo, interpolación de versos y glosas en Met.). Nuestro conocimiento de sendas transmisiones todavía no ha facilitado la articulación de un proceder ecdótico específico y diferenciado, que permita ir más allá de la valoración lingüística y literaria - casi en abstracto- de un sinfín de variantes de apariencia casi equivalente.

A ello se une que el valor de los recentiores es quizá similar - es decir, muy relativo o directamente bajo- en las dos tradiciones que aquí consideramos. $\mathrm{Si}$, como se ha indicado, es remota la posibilidad de que un solo códice, incluso temprano, transmita una lección acertada por vía directa, todavía es más improbable que lo haga un recentior (como ha defendido Tarrant, por citar un solo ejemplo significativo, en el pandis - por blandis- de Met. 10.27165). Según este mismo editor reconocía, hasta Heinsius admitió muchas lecciones de recentiores que eran con frecuencia más bellas o ingeniosas que ciertas ${ }^{66}$.

En ambos textos existe margen, por supuesto, para que un editor actúe como "conservador" 67 o como - dentro siempre de los límites señalados- lo contrario, en un amplio rango de opciones que determinan desde la mera puntuación del texto a la mayor o menor incorporación a éste de conjeturas ${ }^{68}$. Este último aspecto ha llegado a considerarse como el más determinante a la hora de comparar modernas ediciones, pero lo cierto es que tampoco en relación con él se observa una singular audacia o un particular éxito por parte de los más recientes editores de las dos obras aquí analizadas ${ }^{69}$. La entronización de la razón (et ratio et res ipsa, según la célebre conjunción housmaniana) es fácil de compartir como postulado general ${ }^{70}$, pero, en el fondo, quid est ratio? ¿Cuándo debe prevalecer la ops codicum, cuándo la tradición indirecta,

\footnotetext{
${ }^{65}$ Cf., no obstante, el alegato de Tarrant, "Metamorphoses", 277 n. 3, P. Ovidi Nasonis Metamorphoses, XXV-XXVI.

${ }^{66}$ Tarrant, P. Ovidi Nasonis Metamorphoses, XXXII.

${ }^{67}$ Infeliz adjetivo de moda que Tarrant, Galasso y Fedeli dedicaron a Anderson ("Editing Ovid's Metamorphoses", 353, "L'edizione di Richard Tarrant delle Metamorfosi di Ovidio: una discussione", $M D$ 57, 2006, 121, y res. de Tarrant, 606, respectivamente) y Heyworth insinuó respecto al proceder, a su vez, de Tarrant ("The OCT Metamorphoses", 104: "Except in the identification of interpolations, his text is not as different from the Teubner of Anderson $[\ldots]$ as some of us would have hoped, but it is a sound piece of work that will rarely bewilder readers").

${ }^{68}$ D. Butterfield, res. de G. B. Conte, P. Vergilius Maro. Aeneis, Berlín - Nueva York 2009, en ExClass 15, 2011, en 399.

${ }^{69}$ Cf. L. Galasso, "L'edizione di Richard Tarrant”, 121, 132.

${ }^{70}$ Hall, P. Ovidi Nasonis Tristia, XV.
} 
cuándo el usus scribendi o, por el contrario, la anomalía, la excepción o la lectio difficilior? Si, una vez en presencia de dos variantes plausibles, no basta con llegar a señalar una de ellas con el aseado fortasse recte tarrantiano ${ }^{71}$, la investigación del texto debe proseguir y debe hacerlo, siempre que resulte posible, desde la comprensión de los mecanismos internos más específicos de cada transmisión, en la seguridad de que, tarde o temprano, tal conocimiento ayudará al editor a acertar en su elección final o, al menos, le ayudará a justificarla.

Más allá de las concomitancias con la transmisión de Eneida que el propio Ovidio pudo fingir y que sólo afectan en realidad a la prehistoria y primeras fases de su obra más extensa (cuyo conocimiento sigue pendiente de la solución que pueda darse a algunos problemas de carácter textual y, a veces, meramente prosopográfico, como los que hemos señalado en Tristia), los rasgos materiales que diferencian la transmisión textual de Eneida y Metamorfosis son objetivos y numerosos. Se trata de dos tradiciones 'disimétricas' en su origen (de acuerdo con la acertada denominación acuñada o empleada por Jean Irigoin en un trabajo célebre, a propósito de otros dos autores paralelos de enorme envergadura ${ }^{72}$ ); tendieron con el transcurso de los siglos a igualarse, en virtud de mecanismos de transmisión comunes, y generaron finalmente una enorme cantidad de variantes de muy difícil ordenación estemática y que apenas permiten aplicar estrategias de análisis diferenciadas para una y otra obra. Es posible que el texto de Ovidio ofrezca en su conjunto mayor cantidad de variantes y, quizá, menor fijeza editorial que el poema virgiliano, óptimamente constituido, en la práctica, sobre la base de muy pocos testimonios. El estema esbozado para Metamorfosis por Magnus y asumido en lo esencial por Tarrant -instrumento cuya mera existencia también diferencia ambas transmisiones en nuestra práctica más cotidiana- debe seguir sirviendo para autorizar la selección de variantes ovidianas, pese al creciente descrédito que la ratio stemmatica ha ido adquiriendo entre los practicantes de la moderna "corrección" filológica. Y la fijación del texto - muy noble y difícil tarea sin duda ${ }^{73}$ - debe ir precedida de la plena o cabal comprensión de éste, ocupación que algunos editores parecen olvidar a veces, pero que Wilamowitz — nada sospechoso a este respecto- consideraba con toda razón, según testimoniaba Hans von Arnim, como "la más bella tarea de la Filología” ("die schönste Aufgabe der Philologie" 74 ).

${ }^{71}$ Luck, res. de Tarrant, 250, Heyworth, "The OCT Metamorphoses", 107, y, con todo el detalle que ofrece esta reseña en su conjunto, A. Ramírez de Verger, "A new edition of Ovid's Metamorphoses”, en C. Deroux, ed., Studies in Latin literature and Roman history, XIII, Bruselas 2006, 317-18.

${ }^{72}$ Cf. "Deux traditions dissymétriques: Platon et Aristote", en Annuaire du Collège de France, 86 ${ }^{\text {ème }}$ année, 1985-1986, 683-699, y Annuaire..., 87 ème année, 1986-7, 599-615.

${ }^{73}$ Ramírez de Verger, res. de Tarrant, Texts, editors, and readers, 215.

${ }^{74} \mathrm{H}$. von Arnim, Leben und Werke des Dio von Prusa. Mit einer Einleitung: Sophistik, Rhetorik, Philosophie in ihrem Kampf um die Jugendbildung, Berlín 1898 ("Vorwort"). 